\title{
On the Centenary of the Spanish Flu: Being Prepared for the Next Pandemic
}

\author{
William J. Liu' ${ }^{1}$ - Yuhai $\mathrm{Bi}^{2} \cdot$ Dayan Wang ${ }^{1} \cdot$ George F. Gao ${ }^{1,2}$ (B)
}

Received: 23 November 2018/Accepted: 15 December 2018/Published online: 20 December 2018

(c) Wuhan Institute of Virology, CAS 2018

Influenza is one of the oldest infectious diseases affecting humans. Every influenza pandemic in history has ended with disastrous outcomes regarding public health and the social economy. This year marks the 100th anniversary of the Spanish flu (H1N1) outbreak of 1918, which is recognized as the most lethal natural event in recent history. In spite of limited travel and transportation at that time, the Spanish flu pandemic of 1918 reached peak activity on multiple continents simultaneously within several months after its emergence in late 1917 from different hypothesized origins, such as US military camps, the state of Kansas, or the troop staging and hospital camp in Étaples, France (Patterson and Pyle 1991; Oxford et al. 2005; Shanks 2016). However, in some islands of the Pacific region, such as in New Caledonia, the pandemic's lethal effects lasted for over 3 years, until July 1921 (Shanks et al. 2018). The pandemic flu is estimated to have infected more than 500 million people, causing between 50 and 100 million deaths globally (Patterson and Pyle 1991; Guan et al. 2010). The evidence suggests that most pandemicrelated deaths were not caused by the direct pathological effects of the influenza virus, but rather by the lethal effects of secondary bacterial pneumonia (Chien et al. 2009).

In 1957, a pandemic known as the "Asian flu" resulted in millions of deaths caused by a new reassortant influenza $\mathrm{A}(\mathrm{H} 2 \mathrm{~N} 2)$ virus that arose from the circulating human

\footnotetext{
William J. Liu

liujun@ivdc.chinacdc.cn

$\triangle$ George F. Gao

gaofu@ chinacdc.cn

1 Chinese National Influenza Center (CNIC), National Institute for Viral Disease Control and Prevention, Chinese Center for Disease Control and Prevention (China CDC), Beijing 102206, China

2 CAS Key Laboratory of Pathogenic Microbiology and Immunology, Institute of Microbiology, Center for Influenza Research and Early-Warning (CASCIRE), Chinese Academy of Sciences, Beijing 100101, China
}

H1N1 virus with the novel H2, N2, and PB1 genes derived from Eurasian avian viral sources. In 1968, another reassortant influenza virus (H3N2) emerged, causing the "Hong Kong flu". The virus was formed from the backbone of the aforementioned $\mathrm{H} 2 \mathrm{~N} 2$ virus and had the $\mathrm{H} 3$ and PB1 gene segments introduced from the avian flu virus (Guan et al. 2010). "Russian flu", a small but notorious pandemic with H1N1 re-emergence in Russia was reported in 1977. It was found that the genes of this virus were directly derived from the H1N1 viruses circulating in the 1950s (Nakajima et al. 1978). In 2009, a new strain of H1N1 emerged, carrying gene segments derived from swine influenza viruses, causing what was initially called "Mexico Swine flu" (Gao and Sun 2010). Nowadays, the 2009 pandemic virus has replaced the previous seasonal H1N1 viruses that were active for three decades before 2008 and since then has co-circulated in humans along with the influenza $\mathrm{A}(\mathrm{H} 3 \mathrm{~N} 2)$ and influenza $\mathrm{B}$ viruses.

To respond to such unpredictable influenza pandemics in a timely and effectively manner, in 1947, the then newly established World Health Organization (WHO) initiated the Global Influenza Programme (GIP), making influenza the first globally surveilled infectious disease (Fig. 1) (Hay and McCauley 2018). The Global Influenza Surveillance Network (GISN) was established in 1952 as an integrated scientific and technical global collaboration to help the GIP fulfill its objectives and activities. In 2011, the GIP changed its name to Global Influenza Surveillance and Response System (GISRS). The major missions of the GISRS include biannual recommendations on seasonal flu vaccine composition, recommendations on vaccines for potential pandemics, updates on the diagnostic reagents based on the surveillance data, and continuous monitoring of antiviral susceptibility for the evidence of clinical treatment (Hay and McCauley 2018). Such a model platform is needed to achieve global collaboration and timely sharing of viruses, reagents, and information to forestall and respond to public health emergencies, including the potential influenza pandemics. 


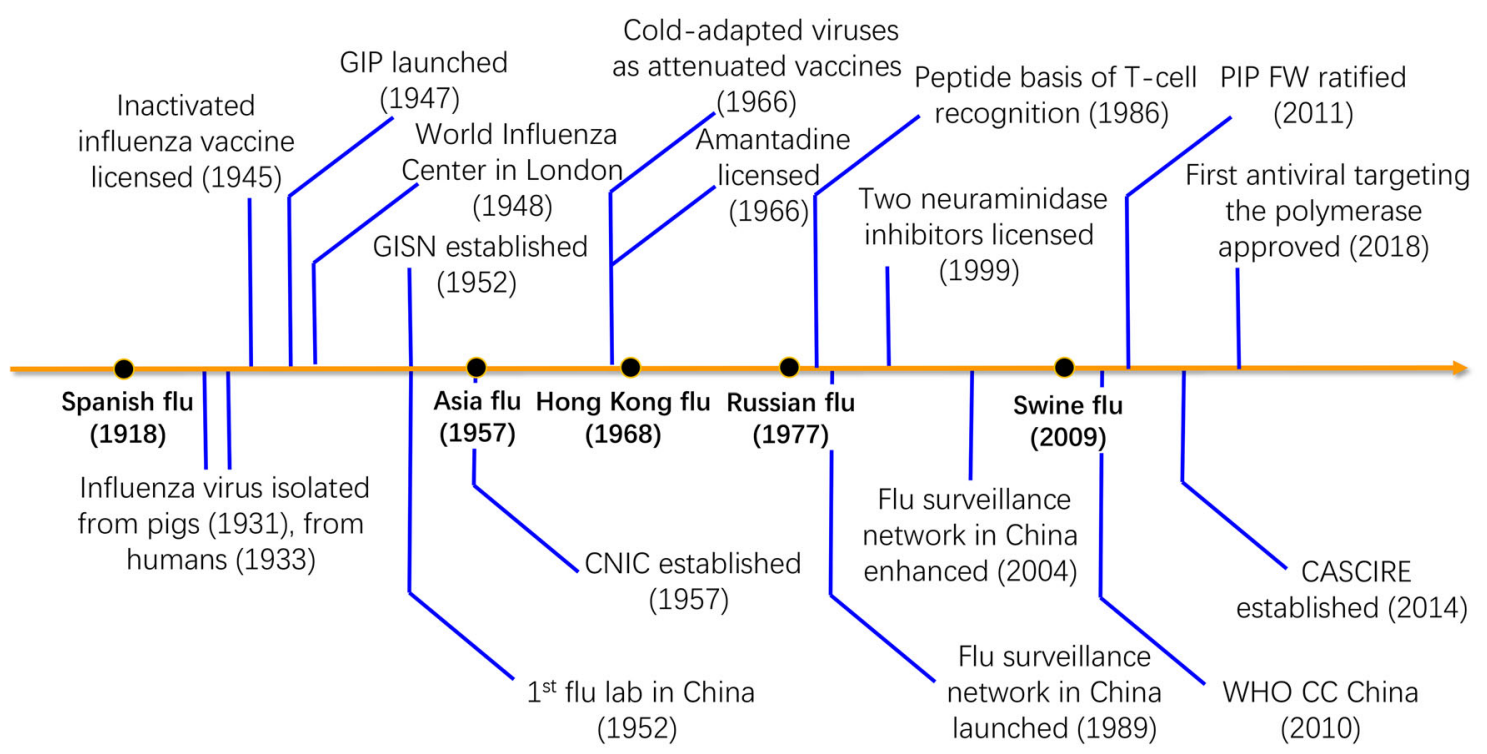

Fig. 1 Milestones of key activities in the influenza control and prevention. The arrow line denotes the time axis with influenza pandemics on it as black nodes. The "Russian flu" in 1977 was also recognized as a "pseudo epidemic". The activities of the WHO and China, and the milestones for the influenza-related studies are shown

As one of the first countries to study influenza viruses, China established the first influenza laboratory-under the guidance of Dr. Chi-Ming Chu in 1952. Later, in 1957 Dr. Chu's group identified the H2N2 subtype responsible for the Asian pandemic, which first appeared in Kweiyang, Kweichow Province, Southern China, and was later recognized as the origin of the "Asian flu" (Yan et al. 2017). The Chinese National Influenza Center (CNIC) was established in 1957 to respond to this first influenza pandemic after the founding of the People's Republic of China. The modern influenza surveillance network was established until 1989 based on six monitoring points, and later strengthened by the Chinese government in 2004 as a response to the high prominence of influenza after the reemergence of the H5N1 virus and the outbreak of the severe acute respiratory syndrome (SARS) (Peiris et al. 2004). After a series of evaluations, in 2010, the CNIC was designated as the fifth WHO Collaborating Center for Reference and Research on Influenza.

Presently, the influenza surveillance network in China is the largest worldwide, covering 408 network laboratories and 554 sentinel hospitals (http://ivdc.chinacdc.cn/cnic/en/). The CNIC provides representative influenza virus isolates to the WHO annually, including about $50 \%$ of the global influenza virus vaccine strains (http://www.who.int/influ enza/vaccines/virus/en/). The Chinese Academy of Sciences (CAS) has also been a backbone force in the fight against influenza since 2005, when the H5N1 subtype was identified among wild birds in Qinghai Lake (Liu et al. 2005), and after a series of other achievements, the CAS above or below the time axis. Abbreviations: GIP, Global Influenza Programme; GISN, Global Influenza Surveillance Network; PIP FW, the Pandemic Influenza Preparedness Framework; CNIC, Chinese National Influenza Center; WHO CC, WHO Collaborating Center for Reference and Research on Influenza.

Center for Influenza Research and Early-Warning (CASCIRE) was established in 2014 (Bi et al. 2017). Together with a series of such professional forces from different institutes, universities and hospitals, the surveillance system for influenza in China was constructed (Han et al. 2017).

A century has passed since the Spanish flu of 1918, but the influenza virus is still relevant today, with an estimated annual morbidity of $10 \%$ in adults and $20 \%-30 \%$ in children infected by seasonal influenza. Of the total cases, 3-5 million are severe and cause between 290,000 and 650,000 result in death (Iuliano et al. 2018). The morbidity and mortality may increase given the constant threat from recent novel zoonotic influenza infections (Gao 2018). Meanwhile, the unpredictable occurrence of influenza pandemics poses a continuous threat to the global public health. Several main strategies would enhance the existing cross-department cooperation strategies and multidisciplinary measures to prevent and control the influenza epidemics/pandemics: global cooperation-based surveillance, development and utilization of vaccines and drugs, and education and promulgation programs for the public.

1. Surveillance is a basic strategy and prerequisite for influenza control and prevention. As one of the global surveillance systems for infectious diseases of the WHO, the GISRS will maintain its leadership in international collaboration on the surveillance of the epidemiology and impact of influenza by sharing viruses for antigenic characterization and selection of 
suitable vaccine viruses to manufacture, regulate, and administrate/distribute influenza vaccines.

2. Although the influenza vaccine is available since 1936 (Plotkin and Plotkin 2018) and ever since vaccination is recognized as one of the best strategies to prevent influenza infection and potential complications after infection, the inoculation rate still needs to improve in developing and underdeveloped countries/regions. Another issue is that the protective effect of currently available influenza vaccines is not as high as most vaccines for other viral diseases, such as measles, polio, and hepatitis B (Osterholm et al. 2012). Furthermore, given the substantial lead time and the lack of available vaccines ahead of any unpredictable pandemic, major efforts are needed to develop new universal vaccines that stimulate broadly reactive antibodies or T-cells against conserved epitopes to confer heterotypic protection against different subtypes of influenza viruses, or at least one subtype in humans or poultries (Xiao et al. 2017; Zhao et al. 2018).

3. More efforts should be paid to the development of new drugs and its corresponding clinical assessment. Amantadine is not recommended anymore due to the broad resistance among $\mathrm{H} 1 \mathrm{~N} 1$ and $\mathrm{H} 3 \mathrm{~N} 2$ viruses. Though oseltamivir can also induce a rapid intra-host emergence of the drug-resistant quasispecies due to the adaptation/evolution of the viruses, it is predominantly recommended in the clinic. Meanwhile, the recent development of the new target-oriented drugs against influenza, such as virus-targeted favipiravir, baloxavir, and pimodivir, and host-targeted DAS181 and nitazoxanide may revolutionize the treatment for influenza.

4. Considering the complexities and particularities of influenza, more attention should be paid to the health education and promulgation for the public. November 1, 2018, marked the first "World Flu Day" that was formally launched at the Asian-Pacific Centenary Spanish 1918-Flu Symposium in Shenzhen, China. The campaign was initiated and developed by Dr. George F. Gao, the Director-General of the Chinese Center for Disease Control and Prevention (CDC), in collaboration with other leading influenza specialists (The Lancet 2018). On the centenary of the Spanish flu of 1918, the "World Flu Day" initiative aimed to raise public awareness about influenza, to accelerate scientific innovation and international cooperation on influenza surveillance, and to push for stronger global support on influenza prevention and control.

Though it is yet a "mission impossible" to predict the next pandemic, it is essential to maintain a global, collaborative defense against influenza. Such globally integrated anti-influenza activity will provide a pre-eminent model for triphibious warfare against other infectious diseases.

Acknowledgements We are grateful to Drs. Zijian Feng, Zijun Wang, Zhongjie Li, Luzhao Feng, and Tao Chen for their excellent suggestions on the preparation of the figure in this manuscript. This work was supported by the National Key Research and Development Program of China (grant 2017YFC1200202), the Major Special Projects for Infectious Disease Research of China (grant 2016ZX10004222003), the National Natural Science Foundation of China (grants 81401312 and 81373141). William J. Liu is supported by the Excellent Young Scientist Program of the NSFC (81822040). George F. Gao is a leading principal investigator of the National Natural Science Foundation of China Innovative Research Group (grant 81621091).

\section{Compliance with Ethical Standards}

Conflict of interest The authors declare that they have no conflict of interest.

Animal and Human Rights Statement This article does not contain any studies with human or animal subjects performed by any of the authors.

\section{References}

Bi Y, Shi W, Chen J, Chen Q, Ma Z, Wong G, Tian W, Yin R, Fu G, Yang Y, Liu WJ, Quan C, Wang Q, He S, Li X, Xia Q, Wang L, Pan Z, Li L, Li H, Xu W, Luo Y, Zeng H, Dai L, Xiao H, Sharshov K, Shestopalov A, Shi Y, Yan J, Li X, Liu Y, Lei F, Liu W, Gao GF (2017) CASCIRE surveillance network and work on avian influenza viruses. Sci China Life Sci 60:1386-1391

Chien YW, Klugman KP, Morens DM (2009) Bacterial pathogens and death during the 1918 influenza pandemic. N Engl J Med 361:2582-2583

Gao GF (2018) From "A"IV to "Z"IKV: attacks from emerging and re-emerging pathogens. Cell 172:1157-1159

Gao GF, Sun Y (2010) It is not just AIV: from avian to swine-origin influenza virus. Sci China Life Sci 53:151-153

Guan Y, Vijaykrishna D, Bahl J, Zhu H, Wang J, Smith GJ (2010) The emergence of pandemic influenza viruses. Protein Cell 1:9-13

Han M, Gu J, Gao GF, Liu WJ (2017) China in action: national strategies to combat against emerging infectious diseases. Sci China Life Sci 60:1383-1385

Hay AJ, McCauley JW (2018) The WHO global influenza surveillance and response system (GISRS) - a future perspective. Influenza Other Respir Viruses. https://doi.org/10.1111/irv. 12565

Iuliano AD, Roguski KM, Chang HH, Muscatello DJ, Palekar R, Tempia S, Cohen C, Gran JM, Schanzer D, Cowling BJ, Wu P, Kyncl J, Ang LW, Park M, Redlberger-Fritz M, Yu H, Espenhain L, Krishnan A, Emukule G, van Asten L, Pereira da Silva S, Aungkulanon S, Buchholz U, Widdowson MA, Bresee JS, Global Seasonal Influenza-associated Mortality Collaborator N (2018) Estimates of global seasonal influenza-associated respiratory mortality: a modelling study. Lancet 391:1285-1300

Liu J, Xiao H, Lei F, Zhu Q, Qin K, Zhang XW, Zhang XL, Zhao D, Wang G, Feng Y, Ma J, Liu W, Wang J, Gao GF (2005) Highly 
pathogenic $\mathrm{H} 5 \mathrm{~N} 1$ influenza virus infection in migratory birds. Science 309:1206

Nakajima K, Desselberger U, Palese P (1978) Recent human influenza A (H1N1) viruses are closely related genetically to strains isolated in 1950. Nature 274:334-339

Osterholm MT, Kelley NS, Sommer A, Belongia EA (2012) Efficacy and effectiveness of influenza vaccines: a systematic review and meta-analysis. Lancet Infect Dis 12:36-44

Oxford JS, Lambkin R, Sefton A, Daniels R, Elliot A, Brown R, Gill D (2005) A hypothesis: the conjunction of soldiers, gas, pigs, ducks, geese and horses in northern France during the Great War provided the conditions for the emergence of the "Spanish" influenza pandemic of 1918-1919. Vaccine 23:940-945

Patterson KD, Pyle GF (1991) The geography and mortality of the 1918 influenza pandemic. Bull Hist Med 65:4-21

Peiris JS, Yu WC, Leung CW, Cheung CY, Ng WF, Nicholls JM, Ng TK, Chan KH, Lai ST, Lim WL, Yuen KY, Guan Y (2004) Reemergence of fatal human influenza A subtype H5N1 disease. Lancet 363:617-619

Plotkin SL, Plotkin SA (2018) A short history of vaccination. In: Plotkin SA, Orenstein WA, Offit PA, Edwards KM (eds) Plotkin's vaccines, 7th edn. Elsevier, Philadelphia, pp 1-15
Shanks GD (2016) No evidence of 1918 influenza pandemic origin in Chinese laborers/soldiers in France. J Chin Med Assoc 79:46-48

Shanks GD, Wilson N, Kippen R, Brundage JF (2018) The unusually diverse mortality patterns in the Pacific region during the 1918-1921 influenza pandemic: reflections at the pandemic's centenary. Lancet Infect Dis 18:e323-e332

The Lancet (2018) World Flu day: momentum from China for influenza control. Lancet 392:1600

Xiao J, Zhang L, Wang Z, Xiang W, Lu P, Zhao Y, Han M, Ma A, Qi P, Wang M, Gao GF, Liu WJ (2017) Conserved peptides enhance immune efficiency of inactive vaccines against emerging avian influenza viruses in chicken. Sci China Life Sci 60:1340-1347

Yan W, Huang B, Ruan L, Tan W (2017) Dr. Chi-Ming Chu: respected founder of molecular virology and pioneer of biologicals in China. Protein Cell 8:629-633

Zhao M, Liu K, Luo J, Tan S, Quan C, Zhang S, Chai Y, Qi J, Li Y, Bi Y, Xiao H, Wong G, Zhou J, Jiang T, Liu W, Yu H, Yan J, Liu Y, Shu Y, Wu G, Wu A, Gao GF, Liu WJ (2018) Heterosubtypic protections against human-infecting avian influenza viruses correlate to biased cross-T-cell responses. MBio. https://doi.org/10.1128/mbio.01408-18 